\title{
Responses to picture-plane and depth mental-rotation stimuli in children and adults
}

\author{
DAVID FOULKES and MICHAEL HOLLIFIELD \\ Georgia Mental Health Institute, Atlanta, Georgia
}

\begin{abstract}
Pairs of toy airplanes with the same or different wings painted red were shown to 12 5-6-yearolds and 10 young adults for judgment of identity as opposed to difference. Figures at the right were rotated, either in the picture plane or in depth, $0^{\circ}, 30^{\circ}, 60^{\circ}, 120^{\circ}$, or $150^{\circ}$ from the position of figures at the left. The data for both picture- and depth-plane rotations revealed a linear relation of reaction time to angle of figural disparity for all adults but only some children. In the case of depth-plane rotations, both the adults and the children had noncontinuous rates of apparent rotation, suggesting other, nonrotational strategies. Child rotators were neither more accurate nor faster than child nonrotators.
\end{abstract}

Research on mental rotation in children was originally initiated to evaluate Piaget and Inhelder's (1966/1971) suggestion that children cannot represent movement in mental imagery until they become (between ages 5 and 8) capable of concrete operational thought. Marmor (1975) found that the rotation phenomenon ${ }^{1}$ occurred in both 5-year-olds and 8-year-olds, and reasoned that this refuted Piaget and Inhelder's claim that kinematic imaging is absent in early childhood. Later, Marmor (1977) reported that she was unable to reject the null hypothesis of rotation-phenomenon differences between children independently evaluated as concrete operational and as preoperational on conservation tasks. However, Kerr, Corbitt, and Jurkovic (1980) studied a larger number of children and found that, in general, conservers exhibited the rotation phenomenon while nonconservers did not. However, their nonconservers were able correctly to identify rotated figures as the same as or different from a standard. Kerr et al. suggested that preoperational children either mentally rotate stimuli in an inconsistent way, or solve rotation problems by nonrotational strategies such as feature analysis, presumably because they cannot perform mental rotation.

Our interest in the rotation phenomenon came from the observation (Foulkes, 1982) that 3-6-year-old children report REM dream imagery that is remarkably static and devoid of physical movement. This observation, along with the data of Kerr et al. (1980), suggests some support for Piaget and Inhelder's account of imagery development, and it seems to refute Mandler's (1983) conclusion that "little evidence is found for qualitatively different kinds of imagery in children and adults (p. 441)." The theoretical significance of the image-quality question for

We appreciate the assistance of Sharon Pearcey, Brenda Sullivan, and Laura Bradley, and the advice of Nancy H. Kerr. Reprint requests should be sent to either author at Georgia Mental Health Institute, 1256 Briarcliff Rd., Atlanta, GA 30329. incremental as opposed to qualitative-change theories of representational development suggested the need for further study of children's ability to generate movement in (or of) mental images and to perform such movement in depth as well as in the picture plane.

\section{METHOD}

\section{Subjects}

The subjects were volunteers recruited through advertising. The young adult subjects were paid for their services, while the children received half of the same sum in money, and half in toys. Ten dextral adults (5 male, 5 female) and 12 dextral 5-6-year-olds (6 male, 6 female, median age 5 years 10 months; age range, 5 years 1 month to 6 years 11 months) were represented in the final data analysis (potential or actual participants not represented in this analysis will be described later).

\section{Mental-Rotation Stimuli}

The stimulus materials were plastic toy airplanes. They were painted red (one wing), white (other wing, vertical tail, top half of fuselage), and blue (horizontal tail, propeller, bottom half of fuselage). The fuselage length was $22 \mathrm{~cm}$, the wing span $28 \mathrm{~cm}$, and the maximum fuselage height $4 \mathrm{~cm}$. During the initial training, these airplanes were mounted on a display apparatus with revolvable metal pins. The apparatus, which was constructed of .8-cm thick fiberboard panels, was $48 \mathrm{~cm}$ deep and $67 \mathrm{~cm}$ wide, with a raised deck $10 \mathrm{~cm}$ above the base and a $67-\mathrm{cm}$-wide backpiece extending $39 \mathrm{~cm}$ above the elevated deck. For depth-plane physical rotation, an airplane was mounted, with a pin through the end of its rear fuselage, $21 \mathrm{~cm}$ from the front and $16 \mathrm{~cm}$ from each side of the apparatus. For picture-plane physical rotation, the same pin holes were used, with the apparatus reversed so that the depth-plane deck became the back and the depth-plane back now lay flush against the table top. Here the airplanes were mounted with a pin perpendicular to the fuselage, through its bottom side just at the rear of the wing section.

The effect of these arrangements was that the subjects saw, at $0^{\circ}$ settings in both the depth-plane and picture-plane series, two airplanes in their line of sight, top-facing and nose up, with rotation possible from top-facing to bottom-facing in the depth plane and from a 12 o'clock position to other clock-face positions in the picture plane. The subjects were exposed to the apparatus itself during training, but they only saw slides of paired airplane configurations on the apparatus during rotation testing. On all trials (apparatus/slide), the left airplane of each pair was portrayed at $0^{\circ}$, while the right airplane could be either at the same setting or rotated from it in the applicable rotational plane by $30^{\circ}, 60^{\circ}$, $120^{\circ}$, or $150^{\circ}$. The subjects' task was to determine whether the two airplanes were the same (same wing red) or different. 


\section{Rotation Training and Pretesting}

The children (but not the adults) were given initial training in discriminating same and different configurations at $0^{\circ}$, both with the apparatus itself and with slides of the apparatus. Following this training, a test was administered. Two four-year-olds failed the same-different pretest.

Following successful same-different discrimination at $0^{\circ}$, the children were given rotation training trials in which rotation of the figures first was physically demonstrated by the experimenter, and then performed by the child. In later rotation training trials, the children were instructed to rotate the "tipped-over" (picture-plane) or "turned-around" (depth-plane) figure "just in your head or mind." Feedback was given on all rotation training trials. There was no criterion pretest with rotated figures.

The adult subjects were told that they were serving as controls for the children. Their rotation "training" series consisted of: exposure to the apparatus; a brief description of the training procedures employed with the children (in which mental rotation was presented as the strategy by which problems were to be solved); and an 8-item pretest with slides. For the children, whose rotation series in each plane was conducted over 2 days, an abbreviated training series was given on the 2 nd day in a condition.

Treatment order (picture- vs. depth-plane) was counterbalanced for both males and females at each age level. When a child moved, on the 3rd testing day, into a new condition, an abbreviated pretest and training series was used. When an adult moved, on the 2 nd testing day, into a new condition, the new plane of rotation was illustrated with the apparatus, followed by an 8-item pretest in that rotation plane.

\section{Rotation Testing}

Picture-plane rotations were clockwise, while depth-plane rotations were with the figure's right wing initiating movement toward the subject. Two points of comparison with stimuli used in earlier research deserve mention. First, our subjects knew that the two stimuli they saw in each trial were structurally identical; the figures varied only in terms of whether one structural component had similar or different coloring. Second, our stimuli permit picture-plane as opposed to depth-plane rotation comparisons in which rotating figures are known to possess identical structural complexity (cf. S. Shepard \& D. Metzler, 1988).

It was explained to the subjects in both groups that their responses were being evaluated for both speed and accuracy. An electronically operated shutter mounted in front of the projector lens of a $35-\mathrm{mm}$ carousel projector controlled exposure times (maximum $10 \mathrm{sec}$ ) and started a digital timer accurate to $.001 \mathrm{sec}$. The timer was stopped when the subject pressed a key on a two-key response box (right key = same, left key $=$ different). The experimenter then read the response-key choice from a lighted display panel and the reaction time from an LED display on the timer. Each test trial was preceded by a verbal preparatory cue. The slides were projected on a blank wall at a distance of $3.3 \mathrm{~m}$; the subjects viewed the slides from a distance of $2.3 \mathrm{~m}$.

The tests consisted of exposing the subjects to identically designed 40-trial blocks of stimulus pairs. In these 40 -trial blocks, a given rightairplane orientation $\left(0^{\circ}, 30^{\circ}, 60^{\circ}, 120^{\circ}, 150^{\circ}\right)$ and left-airplane as opposed to right-airplane red wing configuration (RR, LL, RL, LR) combination occurred once in the first 20 trials and once in the second 20 trials; no single right-airplane orientation was ever present on consecutive trials; as closely as possible, each right-airplane orientation preceded every other one equally often; right-wing orientations nearly matched one another in ordinal position $(M=19.6-21.1)$ and dispersion $(S D=12.0-12.8)$; runs of correct same or different responses never exceeded 3; and runs of correct simple-alternation response patterns (SD or $D S$ ) never exceeded 3. In each condition, the 40-base-trial block was replicated once: in the same test session, following a break, for the adults; in a second test session, for the children (who also were given a break midway through each of their testing sessions).

Overall, then, there were 16 possible data points ( 8 same, 8 different) for each subject for each angle $\left(0^{\circ}, 30^{\circ}, 60^{\circ}, 120^{\circ}, 150^{\circ}\right)$ for each rotation plane (picture, depth). Error RTs were neither repeated until corrected (S. Shepard \& D. Metzler, 1988) nor replaced statistically (Shwartz, 1981). They were simply excluded from analysis. Outlier effects on means were controlled by performing comparisons on median RTs (Wilcoxon tests).

\section{Conservation Testing}

A second experimenter, blind to rotation results, administered Sections A-C (second test session) and D-F (fourth test session) of Goldschmid and Bentler's (1968) standardized conservation battery, Form B, to child subjects only. We defined conservation as a score of 4-6 on the behavioral dimension.

\section{RESULTS}

\section{Adults}

With the exception of an eleventh subject, ${ }^{2}$ not included in the data analysis, the data from all adults exhibited significant $(p<.05)$ linear relationships between angle of rotation and RT for both picture-plane and depth rotations. Median accuracy was $97 \%$ in each condition, and never fell below $90 \%$ for any subject in either condition. Although there were significant linear trends for all 10 subjects, more detailed analysis also revealed a systematic deviation from strict linearity in the depth plane (see Figure 1). There was a group pattern of significantly faster rotation rates (median $556^{\circ} / \mathrm{sec}$ ) between $60^{\circ}$ and $120^{\circ}$ than there was between $30^{\circ}$ and $60^{\circ}$ (median $224^{\circ} / \mathrm{sec}$ ) or $120^{\circ}$ and $150^{\circ}\left(131^{\circ} / \mathrm{sec}\right)$. For neither rotational plane was there a significant RT difference between $0^{\circ}$ and $30^{\circ}$ trials. In both planes, RT differences between subsequent adjacent angular disparities were always significant. However, it was only for picture-plane rotation problems that apparent rotational speed did not differ significantly between adjacent points in the range between $30^{\circ}$ and $150^{\circ}$, the supposed token of a continuous rotation process.

Direct picture-plane versus depth RT comparisons are somewhat ambiguous, since at $0^{\circ}$, where the stimulus displays were almost identical, the depth-plane RT was longer at $\mathrm{p}<.13$ (two-tail). Given this near-significant RT difference, it should cautiously be noted that it was only at $60^{\circ}$ that depth-plane RT was "significantly"

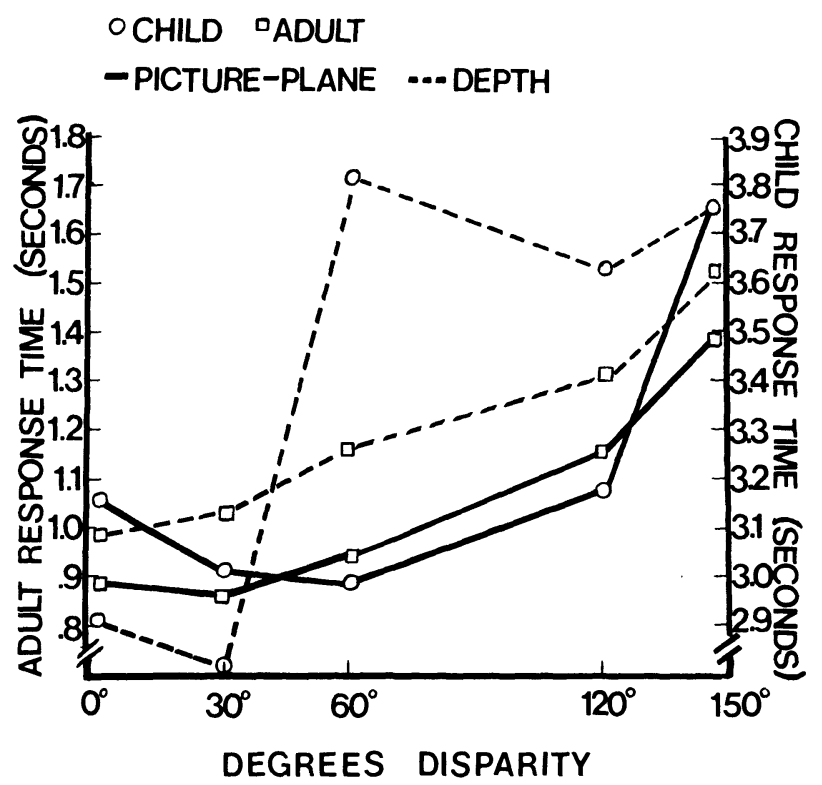

Figure 1. RT by angular disparity. 
greater than picture-plane RT, and that it was only between $30^{\circ}$ and $60^{\circ}$ that the picture-plane rotation rate $\left(429^{\circ} / \mathrm{sec}\right)$ was significantly faster than the depth-plane rotation rate.

\section{Children}

With the exception of a 13th subject (age 4 years 10 months), a chance-level performer on each rotational plane who was not included in the analysis, all the children exceeded chance accuracy in their same-different judgments for each rotational plane. Median accuracy was $99 \%$ for picture-plane rotation and $96 \%$ for depth-plane rotation (n.s.). Although there was more variability in child than in adult accuracy, neither overall, nor at any individual angle of figural rotation in either plane, were children and adults significantly different in accuracy. Thus, we have test problems that young children and adults can solve with equally good accuracy.

As the data plotted in Figure 1 suggest, however, equivalence in accuracy need not imply equivalence in underlying processing. First, as the different numerical legends on the left and right $y$-axes indicate, the children's median RTs for every angle in each plane were significantly and considerably longer than those of the adults. Second, as the raw-data curves suggest, the rotation phenomenon was less evident in the children. ${ }^{3}$ In fact, significant linear relationships between RT and angular disparity were found only for 3 children for both rotational planes, while 5 children exhibited such a relationship for only one plane (picture plane, $n=3$; depth plane, $n=2$ ). Four children did not exhibit the relationship for either plane. Thus, approximately half of the children exhibited the rotation relationship for each plane, but rotation in one plane did not predict the phenomenon in the other plane. Nor did conservation predict the rotation phenomenon. Although the 3 both-plane rotators were all conservers, so too were 2 subjects who exhibited the rotation phenomenon for only one plane and 2 who exhibited it for neither.

As among the adults, with respect to neither plane was there a significant RT difference between $30^{\circ}$ and $0^{\circ}$ trials. However, unlike for adults, comparisons of subsequent adjacent data points were inconsistent. In the picture plane, only the $120^{\circ}-150^{\circ}$ difference was significant. In the depth plane, both $30^{\circ}-60^{\circ}$ and $120^{\circ}-150^{\circ}$ differences were significant, but the median $120^{\circ} \mathrm{RT}$ was shorter than the $60^{\circ}$ RT. In the picture-plane condition, rotation rate did not differ significantly between adjacent angular gaps, but this result is less informative than it was for the adults, all of whom exhibited the rotation phenomenon.

In this light, it is interesting that comparable results were found in depth-plane rotation-rate changes for children and adults. Specifically, the children's apparent rotational speed between $60^{\circ}$ and $120^{\circ}$ was extremely fast $\left(750^{\circ} / \mathrm{sec}\right)$, and significantly faster than the apparent rotation rate between $30^{\circ}$ and $60^{\circ}\left(60^{\circ} / \mathrm{sec}\right)$ and between $120^{\circ}$ and $150^{\circ}$ $(129 \% \mathrm{sec})$.
Also, although the children's baseline $0^{\circ}$ comparison found depth-plane RTs to be insignificantly faster than picture-plane RTs, a reversal of the adult pattern, the same significant rotation-plane differences were found for the children as for the adults: depth-plane RTs longer at $60^{\circ}$, and rotational rate slower between $30^{\circ}$ and $60^{\circ}$ in the depth plane as opposed to the picture plane (where the rotation rate was, in fact, negative: $-196^{\circ} / \mathrm{sec}$ ).

Perhaps the most interesting observations are those relating the rotation phenomenon in children to $R T$ and accuracy. At no angle, in either the picture or depth plane, did either accuracy or RT differ significantly between rotators and nonrotators.

\section{DISCUSSION}

Although the children were predictably slower than adults in solving mental rotation problems, they could solve them equally accurately. Moreover, as Figure 1 indicates, once the intercepts of group data curves on these axes are aligned with one another, subsequent data points fall within a roughly similar area along the $y$-axis. That is, child-adult differences in RT seem to be attributable to processing-time differences (perhaps in encoding and/or response generation) other than those attributable to mental rotation itself.

However, if a mental rotation strategy is indexed by the empirical rotation phenomenon, the children did not reliably (across children) or consistently (across rotation planes) employ such a strategy, and failure to use it affected neither speed nor accuracy of response. From several perspectives, the children's apparent failure reliably and consistently to rotate mental images is understandable. First, several of the children professed, during the training series, not to know what it meant to rotate the figures "just in your head or mind"; two thought that it meant physically rotating the airplanes with their heads. Second, the mental rotation strategy that we and others have attempted to teach children implies deliberate operations performed on conscious mental representations. Observations from attempts to teach such strategies in other areas of research (e.g., rehearsal strategies in verbal learning: Kail, 1984) sug gest that complex, deliberate manipulation of conscious representations is unlikely to occur spontaneously, or to be easily teachable, at ages 5 and 6 . Finally, there must be more humble and unconscious mechanisms by means of which misaligned figures can be compared, mechanisms that serve the adaptive needs of infants as well as other species (Hollard \& Delius, 1982) presumably lacking the sort of reflective access (Pylyshyn, 1978) that mental rotation would require. These mechanisms do not seem to require longer RTs for greater angular disparities.

When the rotation phenomenon is first empirically demonstrable, it may reflect the emergence of some conscious strategy. However, it need not be the particular imaginal strategy described by rotation theorists (e.g., R. N. Shepard \& Cooper, 1982). For our particular version of a depth-plane rotation problem, for example, the nonconstant rate of rotation between $30^{\circ}$ and $150^{\circ}$ suggests something other than a continuous rotation process, particularly since apparent rotational speed is extraordinarily rapid precisely at that juncture where, on a 2-D datastructure hypothesis, stimulus features are most subject to occlusion (Shwartz, 1981). A possible alternative strategy in our depth-plane series is: when the underside of the variable airplane figure is prominent, simply reverse what you directly see (e.g., "if they look the same, they're different"). Problems at $150^{\circ}$ might be somewhat more difficult than those at $120^{\circ}$, because at $150^{\circ}$, as at $30^{\circ}$, the tendency is to make a direct readout, since the rotation of the plane is evident only from its bellyfacing view whereas at $120^{\circ}$ it is evident both from its belly-facing view and from its obliqueness to the line of sight.

A somewhat less sophisticated nonrotational strategy might be: if it seems to be rotated (variable figure at other than $0^{\circ}$ or $30^{\circ}$ ), reverse what you see. Reliance on such a strategy would explain the children's long RTs at $60^{\circ}$, where the strategy is manifestly inappropriate, as well 
as the fact that both the children's and the adults' RT curves differed significantly between depth- and picture-plane only at $60^{\circ}$. We found direct evidence for this unsophisticated label-reversal strategy in the performance at $60^{\circ}$ (depth-plane) of our youngest (5 years 1 month) depthplane (and picture-plane) child rotator: in 16 trials at $60^{\circ}$, there were 15 errors and 1 trial on which no judgment was made within $10 \mathrm{sec}$. However, at $120^{\circ}$ and $150^{\circ}$, where the label-reversal strategy would work, the child had a $69 \%$ accuracy rate. If children can, in fact, produce the rotation phenomenon in the depth plane without engaging in a rotation strategy, then the similar, nonstandard features of their data curves and those of the adults suggest that the rotation phenomenon does not, in our depth-plane test situation, imply rotation strategies at any age level.

Our picture-plane rotation problems were inherently less susceptible to a direct-comparison, label-reversal strategy, since rotations of increasing distance took the variable figure's wings progressively farther away from an alignment affording efficient direct comparison of wing colors. However, the inapplicability of this strategy does not leave mental rotation as the only viable alternative means of solution. It now seems clear that, when faced with rotation problems, subjects may or may not claim to use, and may or may not give the standard empirical evidence for, mental rotation (e.g., see Kaushall \& Parsons, 1981). For children of the ages we have studied here, accurate and efficient solutions clearly are possible without the rotation phenomenon. Although the appearance of the phenomenon may index the first use of some deliberate strategy, that strategy need not be rotational. A wide range of stimuli needs to be investigated in a wide range of contexts before rotation as a process can be inferred from rotation as a phenomenon.

Thus, our data can provide no definitive evidence on the development of kinematic as opposed to static-imagery, or of picture- as opposed to depth-plane data structures (J. Metzler \& R. N. Shepard, 1974; Shwartz, 1981). Yet if there is a shift from representational spatial processing to conscious representational spatial processing (Donaldson, 1978; Liben, 1988) between ages 5 and 8, then systematic research on the rotation phenomenon in this age span may begin to shed some light on the evolution of forms of conscious imaginal experience that are present in mature dreaming, and either that constitute one medium through which adults can solve rotation problems or that increasingly enable children between ages 5 and 8 to access a nonconscious medium through which both children and adults solve rotation problems.

\section{REFERENCES}

Donaldson, M. (1978). Children's minds. London: Croon Helm.

FoulKes, D. (1982). Children's dreams: Longitudinal studies. New York: Wiley.

Goldschmid, M. L., \& Bentler, P. M. (1968). Concept assessment kit-conservation. San Diego, CA: Educational and Industrial Testing Service.

Hollard, V. D., \& DeluUs, J. D. (1982). Rotational invariance in visual pattern recognition by pigeons and humans. Science, 218, 804-806.

KAIL, R. (1984). The development of memory in children (2nd ed.). New York: Freeman.
Kaushall, P., \& Parsons, L. M. (1981). Optical information and practice in the discrimination of 3-D mirror-reflected objects. Perception, 10, 545-562.

Kerr, N. H., CorbitT, R., \& Jurkovic, G. J. (1980). Mental rotation: Is it stage related? Journal of Mental Imagery, 4, 49-56.

LIBEN, L. S. (1988). Conceptual issues in the development of spatial cognition. In J. Stiles-Davis, M. Kritchevsky, \& U. Bellugi (Eds.), Spatial cognition: Brain bases and development (pp. 167-194). Hillsdale, NJ: Erlbaum.

Mandler, J. M. (1983). Representation. In J. H. Flavell \& E. M. Markman (Eds.), Handbook of child psychology: Vol. III. Cognitive development (pp. 420-494). New York: Wiley.

MARMOR, G. S. (1975). Development of kinetic images: When does the child first represent movement in mental images? Cognitive Psychology, 7, 548-559.

MARMOR, G. S. (1977). Mental rotation and number conservation: Are they related? Developmental Psychology, 13, 320-325.

Metzler, J., \& ShEPARD, R. N. (1974). Transformational studies of the internal representation of three-dimensional objects. In R. L. Solso (Ed.), Theories of cognitive psychology: The Loyola symposium (pp. 147-201). Potomac, MD: Erlbaum.

PIAGET, J., \& INHELDER, B. (1971). Mental imagery in the child: A study in the development of imaginal representation. New York: Basic Books. (Original work published 1966)

Pylyshyn, Z. W. (1978). When is an attribution of beliefs justified? Brain \& Behavioral Sciences, 1, 592-593.

ShePARD, R. N., \& COOPER, L. A. (1982). Mental images and their transformations. Cambridge, MA: MIT Press.

SHEPARD, S., \& METZLER, D. (1988). Mental rotation: Effects of dimensionality of objects and type of task. Journal of Experimental Psychology: Human Perception \& Performance, 14, 3-11.

SHWARTZ, S. P. (1981). Three-dimensional mental rotation revisited: Picture-plane rotation is really faster than depth rotation. (Cognitive Science Technical Report No. 9). New Haven, CT: Yale University Cognitive Science Program.

\section{NOTES}

1. We distinguish this empirical rotation phenomenon (a positive, linear relationship between same-different reaction time and the angular disparity of a test figure from a standard) from mental rotation, an inferred form of imagery processing widely held to be responsible for the rotation phenomenon.

2. In picture-plane comparisons only, she had an essentially constant $\mathrm{RT}$ across angle of rotation.

3. Additional analyses indicated that our pooling of same and different data cannot account for the children's failure consistently to demonstrate the rotation phenomenon.

(Manuscript received December 1, 1988.) 